8 December 2016

\title{
Population genetics and demography unite ecology and evolution
}

\author{
Winsor H. Lowe ${ }^{1 *}$, Ryan P. Kovach ${ }^{2}$, and Fred W. Allendorf ${ }^{1}$
}

${ }^{1}$ Division of Biological Sciences, University of Montana, Missoula, MT 59812, USA.

${ }^{2}$ U.S. Geological Survey, Northern Rocky Mountain Science Center, Glacier National

Park, West Glacier, MT 59936, USA.

*Correspondence: winsor.lowe @ umontana.edu (W.H. Lowe) 


\begin{abstract}
The interplay of ecology and evolution has been a rich area of research for decades. A surge of interest in this area was catalyzed by the observation that natural selection can operate at the same contemporary timescales as ecological dynamics. Specifically, recent eco-evolutionary research focuses on how rapid adaptation influences ecology, and vice versa. Evolution by non-adaptive forces also occurs quickly with ecological consequences, but understanding the full scope of ecology-evolution interactions requires explicitly addressing population-level processes - genetic and demographic. We show the strong ecological effects of non-adaptive evolutionary forces and, more broadly, the value of population-level research for gaining mechanistic understanding of ecologyevolution interactions. The breadth of eco-evolutionary research should expand to incorporate the breadth of evolution itself.
\end{abstract}

Keywords: demography; genetics; dispersal; genetic drift; density dependence; natural selection

\title{
The Scope of Ecology-Evolution Interactions
}

Ecological and evolutionary processes influence all levels of biological organization, but ecology and evolution are inseparable at the population level. Survival, recruitment, abundance, density, and exchange of individuals among populations are all determined by the interaction of ecological and evolutionary processes $[1,2]$. In turn, these 
demographic parameters determine whether and how variation in individual fitness is converted into higher-order ecological and evolutionary effects, setting the rate and scale of future ecology-evolution interactions [3-5].

Population genetics theory provides a strong framework for understanding the interdependence of ecological and evolutionary processes by accounting for effects of demography on phenotype and gene frequencies (Figure 1). Natural selection acts at the level of the individual, but both adaptive and non-adaptive evolutionary forces acting at the population level determine whether, how, and at what spatial scale phenotypic change and resulting ecological consequences occur [6,7]. Likewise, ecological dynamics acting at higher levels of organization (e.g., species interactions in communities) must affect population processes to drive adaptive and non-adaptive evolution [8,9], whether through changes in selection, genetic drift (see Glossary), or gene flow.

In the last decade, ecologists have embraced the concept of eco-evolutionary dynamics, which emphasizes the power of ecological selection to cause rapid adaptation and, likewise, for adaptive evolution to influence ecological processes in real time [10,11]. The perceived novelty of this concept appears to stem from the fast rate of interaction between ecological conditions and phenotypic adaptation, which contrasts with traditional, gradualistic models of adaptation. The eco-evolutionary dynamics concept is challenging, including the difficulty of characterizing the rate of ecology-evolution interaction in a way that is not biased by human perception, and the fact that all adaptive (or non-adaptive) evolution can be linked to ecological causes and consequences. 
However, there is also no doubt that exciting examples of observable ecology-evolution interactions have come to light recently [12-14], adding to a foundation of classic examples [15-18].

We are mainly concerned that a narrow focus on adaptation in eco-evolutionary studies obscures equally strong and rapid ecological effects of non-adaptive evolutionary forces (Figure 1). To understand the ecological causes and consequences of the full scope of evolutionary forces, eco-evolutionary research could benefit from explicit integration of population processes - genetic and demographic. Greater integration of population-level processes will not only elucidate the ecological effects of non-adaptive evolution, but also provide novel insight on how these non-adaptive forces promote (or prevent) the rapid phenotypic adaptation currently spotlighted in this research.

Our goal is to show the importance of considering the full scope of evolutionary processes - adaptive and non-adaptive - to advance mechanistic understanding of ecology-evolution interactions. We first review the treatment of population genetic principles in evolutionary biology and ecology, showing the history of a view that population-level processes conflict with adaptive evolution - a misperception that still influences eco-evolutionary research, implicitly and explicitly. To move beyond this view, we then review studies showing how demographic parameters mediate ecologyevolution interactions via adaptive and non-adaptive mechanisms. Finally, we identify emerging opportunities for population-level research on the interplay of ecology and evolution. 


\section{Wrestling with the Population Context of Ecology-Evolution}

\section{Interactions}

Population genetics theory provides a mechanistic framework for integrating ecological and evolutionary processes by explicitly incorporating population parameters that are, themselves, shaped by ecological interactions (Box 1). More generally, population genetics theory illuminates the full suite of forces that promote and prevent evolutionary change, and the genetic and demographic mechanisms that govern these forces. In contrast, the recent surge of eco-evolutionary research hinges on the rate of adaptive phenotypic change, thus emphasizing directional selection at the cost of other evolutionary processes and their demographic bases. While often described as "contemporary evolution", this area of research might be better described as "contemporary adaptation" [19].

The rift between long-standing population genetics theory and current eco-evolutionary research underscores the challenge of fully addressing the forces that drive evolution at the population level. This challenge is not new [20; p. 64], and is embodied in a historical debate over the power of selection to drive evolutionary change in the face of other, nonadaptive forces - a debate that is largely settled in the field of evolutionary biology, but the root of a narrow view of evolution in many current eco-evolutionary studies. 
By treating genes as independent units, population genetics was criticized by some evolutionary biologists as overly simplistic and devoid of the functional interrelationships among genes that are crucial to selection [reviewed in 21]. Mayr [22] questioned whether this "mathematical" school contributed anything to evolutionary theory, characterizing gene pool models as "beanbag genetics" because they merely track the frequency and distribution of individual genes, ignoring the functional integration of these genes. This criticism reflects a broader debate over the relative power of selection to drive evolutionary change.

Debate over hard v. soft selection also centers on the power of selection to produce rapid adaptive change. Under hard selection, genotypes with fitness below a threshold are removed from the population [23]. This process can result in the rapid adaptive change spotlighted in current eco-evolutionary studies. Under soft selection, the availability of different niches in the environment and competition among genotypes results in adaptive change [24]. There are no optimal genotypes, but rather a range of successful genotypes determined by the combination of extrinsic forces, density, and the frequency of other genotypes in the population. Soft selection presents a model of adaptive evolution that can maintain a range of successful phenotypes, and high standing genetic variation [25]. Assessing hard v. soft selection requires population-level analyses of genotype and phenotype frequencies and densities. Interestingly, a recent review suggests that cases of rapid adaptive evolution highlighted in the eco-evolutionary dynamics literature require high amounts of standing genetic variation [26], showing the importance of understanding forces (like soft selection) that maintain that standing variation. 
The quasi-instantaneous interplay of ecology and adaptation that has come to define ecoevolutionary dynamics is consistent with a traditional focus on unencumbered adaptive evolution among ecologists. In 1969, Ehrlich and Raven [27] argued that "selection is both the primary cohesive and disruptive force in evolution, and...the selective regime itself determines what influence gene flow (or isolation) will have." Their argument centers on the relative influence of selection v. gene flow, but their assertion of the primacy of selection implies equally weak effects of the other non-adaptive evolutionary processes (Figure 1).

Acknowledgement of the complexities of evolution at the population level can be traced through subsequent decades in the ecological literature [28,29]. Nevertheless, there remains an overarching view that strong ecological effects are predominantly associated with local adaptation [30,31]. The limitations of this view are made evident by strong ecological effects of non-adaptive evolution. This view also obscures the great value of population-level research for uncovering the broader implications of ecology-evolution interactions [e.g., 32,33], including what determines the rate of these interactions and the spatial scale of their influence.

\section{Research at the Population Ecology-Evolution Interface}

Ecology-evolution interactions occur in diverse systems and affect multiple levels of ecological organization [11]. The next challenge is to gain insight on the mechanisms that 
determine the spatial scope and strength of these "real-time" feedbacks. This will require explicitly addressing the role of population processes (Figure 2), which mechanistically unite ecological and evolutionary dynamics across levels of response. Below we review studies showing these evolution-demography links, and thus offering a roadmap to improve integration of population genetic and demographic principles in ecoevolutionary research.

\section{Population Size}

Perhaps the most direct interaction of genetics and demography results from the effect of population size on population viability and associated ecological consequences. One of the oldest empirical observations of population genetics is that offspring of related individuals tend to have lower fitness than offspring between unrelated individuals [34]. The "inbreeding effect of small populations" leads to reduced fitness of individuals in small populations primarily because of increased homozygosity for deleterious recessive alleles [9, p. 101]. However, it has been somewhat difficult to determine the effects of decreased fitness of individuals due to inbreeding on population demography and resulting ecological interactions.

Johnson et al. [35] modeled the demographic consequences of inbreeding depression in bighorn sheep (Ovis canadensis). Their approach reduces mean vital rates of cohorts rather than modeling the individual effects of inbreeding depression, yet these individual effects are likely to have important demographic and ecological implications [36,37]. Specifically, Johnson et al. [35] use a female-based model that does not incorporate the 
effects of inbreeding depression on one-half of the population (i.e., males). Also, this approach reduces the fecundity of all females equally rather than taking into account that the fitness of most individuals (i.e., those that are not inbred) will not be reduced. Because the relationship between reduction in fitness and inbreeding coefficient is not linear [38], using the mean inbreeding coefficient will underestimate the mean reduction in fitness. Finally, inbreeding depression affects many aspects of fitness not included in this model [39].

Johnson et al. [35] projected the demographic influence of inbreeding depression in future generations by increasing the mean inbreeding coefficient of the population based on estimates of effective population size $\left(\boldsymbol{N}_{\mathrm{e}}\right)$ and increasing the mean inbreeding coefficients of individuals by $1 / 2 N_{\mathrm{e}}$ per generation. This rate of increase assumes selective neutrality (i.e., no inbreeding depression), but we know that differences among individuals in the proportion of their genes identical by descent (IBD) because of inbreeding provide an opportunity for natural selection to eliminate individuals with a greater proportion of the genome IBD. There can be great variability in the proportion of the genome IBD, even among individuals with the same inbreeding coefficient [40]. For example, Bensch et al. [41] found in a population of wolves (Canis lupus) that the most heterozygous wolves for each value of the pedigree inbreeding coefficient became established breeders. This selection process reduced the loss of heterozygosity despite a steady increase of the inbreeding coefficient. 
Studies of the livebearing topminnow Poeciliopsis monacha show the demographic context of inbreeding in small populations and subsequent genetic rescue [42]. This fish inhabits rocky arroyos in northwestern Mexico. The upstream portion of a small stream dried completely in 1976, but within 2 years fish recolonized this area from permanent springs downstream. Fitness of the source and founder populations was compared with that of coexisting asexual forms of Poeciliopsis that experienced the same extinctionrecolonization event. Cloning preserves heterozygosity, eliminating inbreeding depression in the asexual fish.

Compared to clones, the inbred founder population of $P$. monacha exhibited reduced fitness and an increased parasite load [43]. By 1983, P. monacha was nearly eliminated from several small pools while the clones flourished. The founder population was then rescued by transplanting 30 genetically variable females from a downstream location where $P$. monacha was genetically variable. By the following spring (2-3 generations), sexual P. monacha regained numerical dominance over the clones [42], and its parasite load dropped to amounts that were typical of the permanent localities downstream [43].

A growing number of cases of genetic rescue [reviewed in 44] provide vivid - but often overlooked - evidence of strong and rapid ecological effects of non-adaptive evolutionary processes, where the presence of a species and its web of biotic and abiotic effects depend on the restoration of genetic variation lost at small population sizes. In a striking example, population dynamics of wolves on Isle Royale, USA - and the numerous ecological effects of this apex predator - show the harmful effects of 
inbreeding and the positive effects of gene flow [45]. Genetically driven cycles in the population dynamics of this large carnivore have far-reaching ecological consequences; wolves on Isle Royale directly influence moose population dynamics and behavior, and thus indirectly influence community composition through trophic cascades mediated by moose herbivory [46].

Small population size will also lead to the loss of allelic diversity [47], which can have strong demographic and ecological effects even in the absence of inbreeding depression. Many flowering plant species have genetic incompatibility mechanisms. In one system, an individual's mating type is determined by its genotype at the self-incompatibility $(S)$ locus [48]. Pollen grains can only fertilize plants that do not have the same $S$-allele as the pollen, and smaller populations are expected to maintain many fewer $S$-alleles than larger populations at equilibrium [49]. Reinartz and Les [50] concluded that some one-third of the remaining 14 natural populations of Aster furactus in Wisconsin had reduced seed sets because of a diminished number of $S$-alleles. An analogous effect occurs in the nearly $15 \%$ of animal species that are haplodiploid, in which sex is determined by genotypes at one or more hypervariable loci [51,52].

\section{Population Density}

Most populations are influenced by density regulation [53], but integrating evolutionary processes into population models with density dependence has been a challenge of evolutionary ecology for decades $[4,5]$. The influence of density on fitness and ecologyevolution interactions continues to be of interest, especially in relation to life history 
evolution [54] and dispersal [30]. The strength of density dependence also plays a key role in determining the form of natural selection acting on phenotype and allele frequencies [hard v. soft selection; 24], which in turn influences population dynamics [2].

Soft selection is a density dependent (and frequency dependent) process closely tied to population dynamics. For example, soft selection can reduce variation in population abundance - a fundamental ecological response - when limiting factors compensate for the demographic costs of hard selection, that is when soft selection results in compensatory mortality. The demographic context of selection is exemplified by research showing the desynchronization of egg laying in great tits (Parus major) and availability of caterpillar prey [55]. Spring warming has increased phenological mismatch between reproduction in great tits and the caterpillar food resource, increasing directional selection for earlier egg laying. However, demographic costs of selection are currently offset by relaxed competition for food and breeding territories among young birds. Such compensatory dynamics might be necessary for species to persist while undergoing rapid adaptive evolution [56], but detecting these dynamics requires population-level analyses.

Density dependence influences other ecology-evolution interactions with relevance to conservation. In high-density populations, disruptive selection resulting from intraspecific competition can lead to life-history variation that simultaneously increases genetic and phenotypic variation, population productivity, and population stability [57]. This increase in standing genetic variation increases the maximum potential rate of evolution due to natural selection [58]. Greater phenotypic variability can also increase 
effective population size directly or by reducing variation in $N_{\mathrm{e}}$ across generations [59]. Likewise, populations at low densities due to human harvest can have reduced adaptive variation owing to relaxation of intraspecific competition and diversifying selection, loss of variation through drift or anthropogenic selection, and reduced opportunity for the stabilizing demographic effects of soft selection. Together, these factors can reduce the efficacy of evolution by natural selection and threaten long-term population persistence [60].

\section{Population Connectivity}

Dispersal and gene flow are commonly seen as threats to local adaption, thus limiting the strength of ecology-evolution interactions [27,31]. This view is based on the assumption that selective regimes differ between source and recipient populations such that dispersal introduces genes mismatched to the selective regime of the recipient population [61]. Even under this assumption, gene flow can interact with local selection regimes to produce a range of ecological effects and adaptive consequences (Box 2) [62]. Furthermore, recognition that dispersal is not an entirely random process has led to a more nuanced understanding of its ecological and evolutionary implications $[63,64]$.

Recent work on animal personalities illustrates the ecological and evolutionary effects of non-random dispersal, and the role of population processes in mediating these effects. Animal personality has repeatedly been linked to dispersal propensity, with individuals exhibiting "bold" or "exploratory" personalities more likely to disperse than those with "shy" personalities [65]. These personality traits often affect local ecological dynamics 
[66] and we are beginning to uncover their genomic basis [67]. In combination, these attributes of animal personality - close association with dispersal, strong ecological effects, and genetic basis - set the stage for rapid and spatially extensive ecologyevolution interactions.

A genetic correlation between dispersal and aggression enables western bluebirds (Sialia mexicana) to invade and displace less-aggressive mountain bluebird (S. currucoides) populations in the northwestern U.S.A., influencing landscape-level patterns of distribution and abundance in both species [68]. Following displacement of mountain bluebirds, population-level feedbacks determine temporal patterns of phenotypic change and abundance in western bluebirds. Specifically, population size and frequency of the aggressive phenotype are driven by density-dependent demographic effects (i.e., reduced fitness of aggressive individuals at high densities) and maternal effects (i.e., increased production of aggressive offspring at high densities).

A similar association among dispersal, personality, and ecological effects occurs in the spider Anelosimus studiosus. Docile and aggressive phenotypes are heritable, the aggressive phenotype is associated with increased exploratory behavior and dispersal distance, and the species builds webs that serve as habitat for $>50$ other species of spider [69]. In a 7-year study of experimentally-established A. studiosus colonies, the personality of founding individuals affected successional patterns of species composition and community longevity [70]. Furthermore, variation in the docile:aggressive ratios of 
A. studiosus populations appears to be related to site-specific selection at the group level, where colony survival depends on the optimal docile:aggressive ratio [71].

Strong association with dispersal and local ecological interactions is not restricted to personality traits $[66,72]$. However, these examples underscore the potential for spatially extensive ecology-evolution interactions, where genetically based traits of dispersing individuals exert strong ecological effects in recipient populations. They also show how traits of dispersers can influence the rate of adaptive evolution in recipient populations depending on how dispersal phenotypes match the selective regime of a recipient population (e.g., competition with mountain bluebirds). A similar situation can occur under the non-equilibrium conditions of species invasion, where the best dispersers at the invasion front also have traits that allow establishment [73]. These examples show the

critical importance of quantifying population parameters (e.g., dispersal, gene flow, local population dynamics) to understand the true spatial scale at which ecology-evolution interactions are occurring and their demographic and community-level implications $[74,75]$.

\section{Future Directions}

Novel methods in population genomics and demographic estimation will create exciting opportunities to advance mechanistic understanding of ecology-evolution interactions. But, we must first acknowledge the advances already resulting from modeling approaches that integrate population-level ecological and evolutionary processes. Coulson et al. [76] 
developed a framework that links quantitative genetics and structured population modeling to capture the population-level dynamics of phenotypes controlled by additive genetic components. This flexible framework allows the simulation of a wide range of feedbacks between ecological and evolutionary variables, including population growth rates, life history, heritability, genetic variance-covariance structure, and adaptation. Using integral projection models and simulations based on field data, Rees and Ellner [32] show that changes in population structure (e.g., size or stage) can strongly affect overall selection pressure, in addition to the changes in demography (i.e., growth, survival, fecundity) commonly associated with selection.

The genomics revolution has given us unprecedented opportunities to explore how genome composition and structure influence ecological function at the level of the whole organism [77]. However, this depth of focus often comes at the cost of a broader perspective on how genomic variation is partitioned among individuals within populations, and among populations linked by gene flow. Recent studies showing individual-level genomic changes with clear population-level ecological implications show the importance of closing this gap. For example, Myburg et al. [78] genotyped 28 progeny from a single Eucalyptus grandis tree at 9,590 loci. Fifty percent of the genome is expected to be IBD in progeny produced by selfing. However, the 28 progeny lost an average of just $34 \%$ of heterozygosity (range $=21 \%-48 \%$ ) compared to the parental tree. Results such as these provide new opportunities to understand the genetic basis of inbreeding depression so that it can be incorporated into population models. 
Advances in genomic resolution are even more exciting in light of parallel advances in methods for quantifying demographic rates in wild populations. Spatial capture-markrecapture methods can now quantify demographic rates within populations (i.e., survival and recruitment) and rates of dispersal among populations (i.e., immigration and emigration) [79]. In combination with population genomic analyses, estimates of local and spatial demographic rates can resolve cryptic interactions between gene flow and selection (Figure 2). For example, it is now possible to compare genetic divergence to population-specific immigration and emigration rates [80].

The combination of genomic and demographic tools can also provide new insight on the evolutionary basis and ecological effects of intraspecific phenotypic variation [36,81]. Much of the interest in ecology-evolution interactions seems to stem from observations of intraspecific covariation in phenotype and ecology. Traditionally, assessing environmental v. genetic influences on this phenotypic variation has required transplant experiments or intensive pedigree analyses. By taking advantage of the high resolution of genomic markers, it is now possible to assess genetic structure associated with specific phenotypes, both within and among populations [82]. Current capture-mark-recapture methods offer the same resolution of rates of survival, recruitment, and dispersal among phenotypes [83]. With these methods, it is possible to test for concordance between genomic and demographic estimates of the strength and type of selection acting on phenotypes [84], and to track the ecological and genetic effects of dispersal-associated phenotypes [85]. 
In experimental studies of ecology-evolution interactions, we see great value in treatments that vary demographic and genetic parameters in addition to selection (Figure 1B). To date, most eco-evolutionary experiments have applied ecological treatments to replicate 'populations', which are then monitored for adaptive response. This approach typically standardizes the size, composition (e.g., genetic variation, stage or size structure), and connectivity of populations - all of which will influence evolutionary response. Adding treatments that manipulate these population parameters will increase the applicability of results to natural systems, and reveal interactions between adaptive and non-adaptive evolutionary processes [86,87].

\section{Concluding Remarks}

This review aims to promote interdisciplinary research by highlighting the inseparability of ecological and evolutionary processes at the population level, thus setting a common platform for integrative studies. The struggle to understand evolution at the population level - and associated ecological causes and consequences - is not new. Despite this history, our excitement at the power of natural selection to produce rapid adaptation and ecological responses often eclipses fundamental questions of how adaptive evolution occurs in complex natural systems and - as importantly - the ecological causes and consequences of non-adaptive evolution. Population-based approaches that explicitly integrate genetic and demographic information are necessary if we hope to gain insight on the full scope of mechanisms driving ecology-evolution interactions. 


\section{Acknowledgments}

We thank Dave Tallmon, Mike Lynch, Tim Coulson, Rolf Peterson, John Vucetich, and Kara Cromwell for their help and advice, and four anonymous reviewers for detailed comments that improved this manuscript. Funding comes from NSF grants DEB1050459 and DEB-1258203. RPK was funded by a USGS Mendenhall Fellowship. Any use of trade, firm, or product names is for descriptive purposes only and does not imply endorsement by the U.S. Government. 


\section{Figure Legends}

Figure 1. Evolutionary and Ecological Processes are Inseparable. Conceptual illustration of interconnections among evolutionary forces and ecological interactions (biotic and abiotic) through population-level demographic and genetic parameters. (A) represents those interconnections emphasized in current eco-evolutionary research. (B) represents a more comprehensive model of these interconnections, including the full suite of evolutionary forces and a range of population parameters that are themselves interdependent. We build our review around population demographic parameters (size, density, connectivity), but describe key interactions with genetic parameters (mean fitness, genetic variation). We define mean fitness according to population genetics theory, as the sum of the fitnesses of genotypes in a population weighted by their proportions [88], thus representing the population-level effects of local adaptation.

\section{Figure 2. Population Parameters Affect the Balance of Adaptive and Non-Adaptive} Evolutionary Forces. Illustration of the relative influence of different evolutionary forces in a population exposed to predation (A) as a function of population size (B), population density (C), and population connectivity (D). Text size represents variation in the relative influence of each evolutionary force. 
(A)

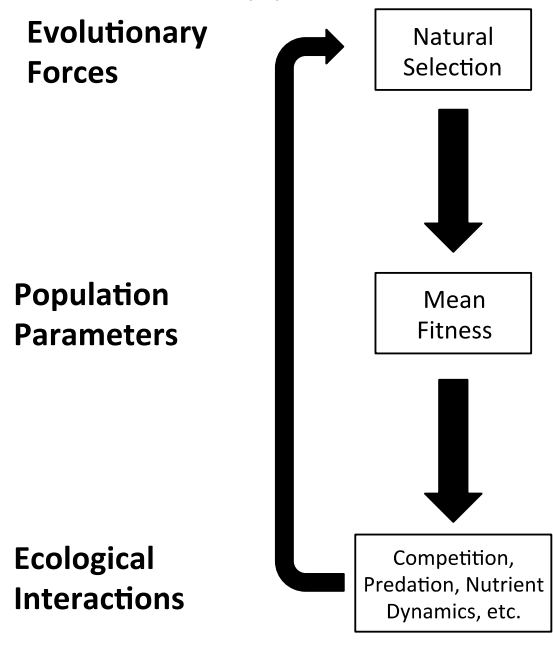

(B)

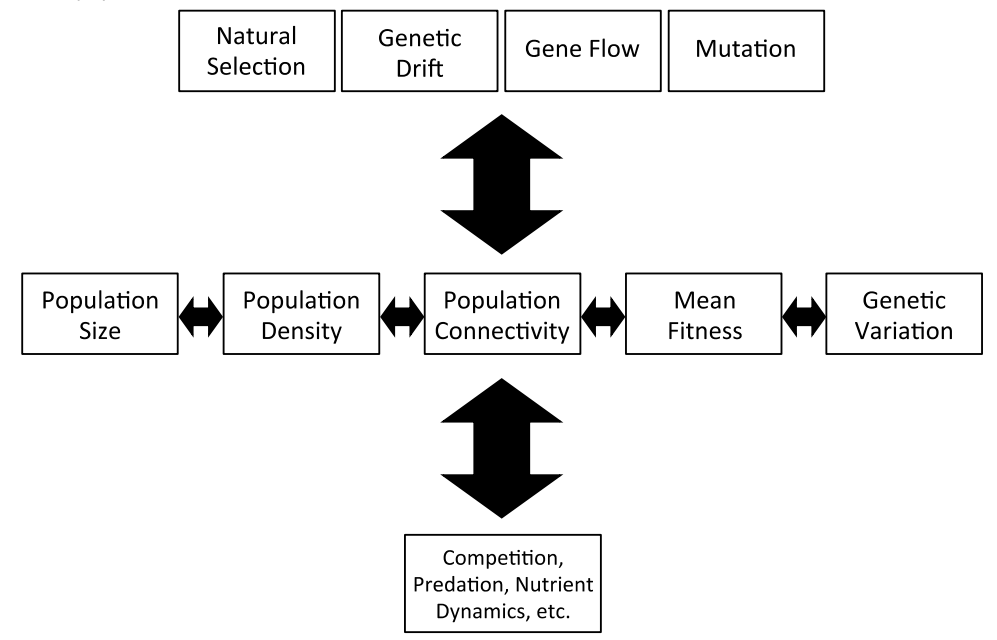

Figure 1 


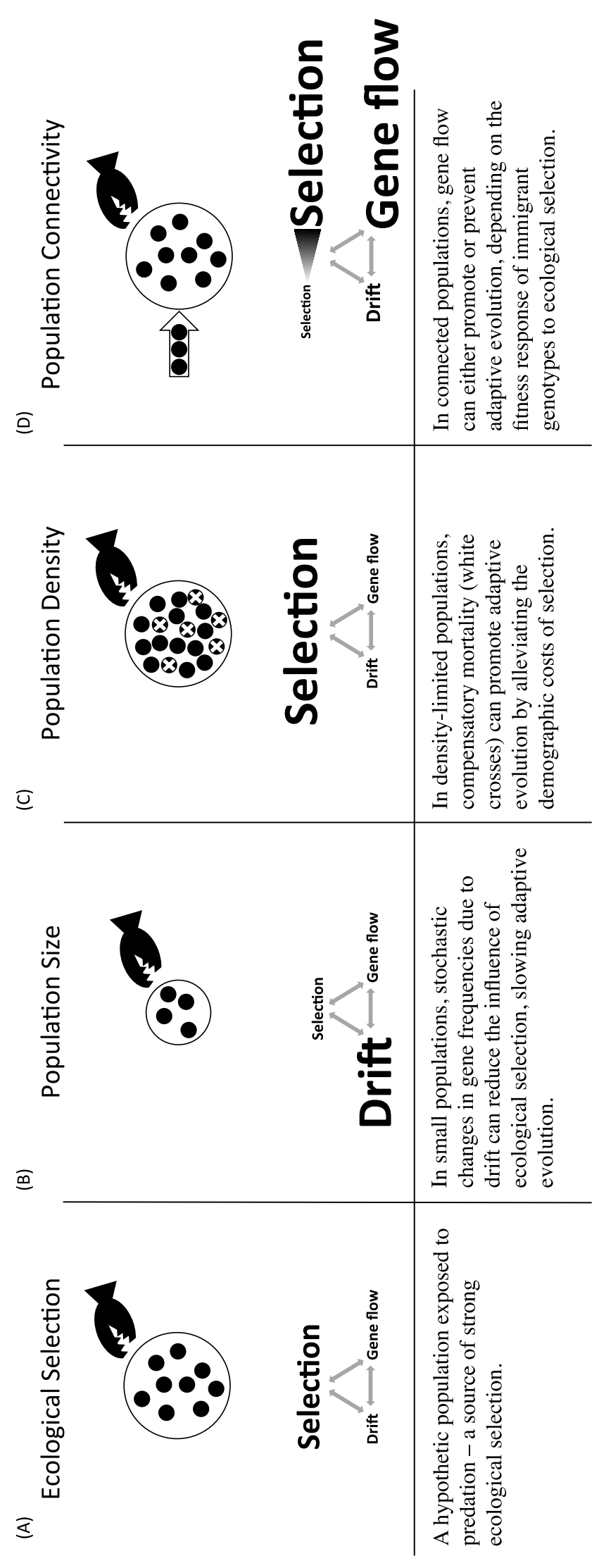

Figure 2 


\title{
Text Boxes
}

\section{Box 1: Interactions between Population Genetic and Demographic Processes}

\author{
"The fusion of population genetics with population ecology \\ can be compared to a prearranged marriage between partners \\ who speak different languages."
}

Roughgarden (1996)

There are four fundamental population genetic processes that interact with population demography: natural selection, genetic drift, mutation, and gene flow. Here we consider the interaction of the first three of these processes with demography. The effects of gene flow are considered in detail in Box 2.

Natural selection brings about local adaptation that results in increased fitness of individuals in their local habitat. Nevertheless, trait changes by natural selection that are advantageous to individuals do not necessarily benefit the population. Haldane [89; pp. 65-70] suggested that traits can evolve by natural selection that are detrimental to the population. The concept that natural selection can decrease the viability of a local population has been called "evolutionary suicide" [90]. 
Genetic drift causes stochastic fluctuations in allele frequencies resulting from random differences in the survival and fecundity of individuals, as well as binomial sampling of alleles during meiosis. These stochastic effects can overwhelm the effects of selection in small populations. Thus, smaller populations are less likely to become locally adapted. Johansson et al. [91] found reduced local adaptation in small isolated populations compared to continuous large populations of the common frog (Rana temporaria). They concluded that the effects of genetic drift swamped the effects of natural selection in smaller populations.

The relevant metric of population size here is the genetically effective population size $\left(N_{\mathrm{e}}\right)$, not the census size. In general, changes in allele frequency are determined primarily by genetic drift rather than by natural selection when the product of $N_{\mathrm{e}}$ and the selection coefficient is less than one [92]. Thus, a deleterious allele that reduces fitness by, say, 5\% will act as if it were selectively neutral in a population with $N_{\mathrm{e}}$ of 20 . The increase in frequency of deleterious alleles in small populations (inbreeding effect of small populations) can decrease the viability of small populations. Under Wright's shifting balance theory, random genetic drift allows subpopulations to explore the adaptive landscape and potentially occupy a higher adaptive peak [93].

Mutation is a change in DNA sequence or chromosome arrangement in the transmission of genetic information from parent to progeny. Mutation is the ultimate source of advantageous alleles. However, mutation rates are generally extremely low (on the order of $10^{-6}$ per locus), and thus have demographic effects only over the very long-term. 
Advantageous mutations could be more likely to be incorporated into large than small populations because more mutations will occur and selection is more effective in larger populations. In contrast, Wright [94; p. 157] first suggested that small populations would decline in vigor slowly over time because of the accumulation of deleterious mutations that natural selection would not be effective in removing, because of the overpowering effects of genetic drift. As deleterious mutations accumulate, population size might decrease further and thereby accelerate the rate of accumulation of deleterious mutations. This feedback process has been termed mutational meltdown [95]. 


\section{Box 2: The Nuances of Gene Flow}

Long-standing population genetics theory and recent empirical work show that not all amounts of gene flow will have homogenizing effects on populations, and thereby work against local adaptation [Figure IA; 62,80]. One immigrant per generation ensures that the same alleles will be shared among populations over long periods of evolutionary time [96], but does not preclude considerable genetic differentiation (i.e., high $\boldsymbol{F}_{\mathbf{S T}}$ ) among subpopulations [88]. Subpopulations are expected to have equal allele frequencies only if they are panmictic, which requires considerably more exchange than one migrant per generation [ $\geq 10$ individuals per generation; 97]. In contrast, even small amounts of gene flow (an average of one immigrant every 10 generations) are sufficient to allow advantageous alleles to spread across populations [98].

In a population exposed to strong directional selection, effective population size $\left(N_{\mathrm{e}}\right)$ also influences the relationship between gene flow (migration, $m$ ) and mean fitness (Figure IB). Realized mean fitness is a function of both inbreeding depression and local adaptation. Isolation can result in reduced fitness because of the accumulation of inbreeding depression in small, isolated populations. This effect is alleviated by at least one migrant per generation $\left(N_{\mathrm{e}} m=1\right)$. Thus, smaller values of $m$ are required to alleviate the effects of inbreeding depression in larger populations. Genetic drift also reduces the efficacy of natural selection in small populations. Thus, larger populations are expected to show greater mean fitness. Finally, local adaptation can be swamped at large migration rates, resulting in lower fitness at high values of $m$. 
These complex relationships among gene flow, genetic variation, and adaptation underscore the value of direct measurements of dispersal and local demographic parameters to understand ecology-evolution interactions.

Figure IA. Three types of genetic connectivity. Expected values of $F_{\mathrm{ST}}$ with the island model of migration [99]. Arrows indicate the $F_{\mathrm{ST}}$ values for the three types of genetic connectivity - the degree to which gene flow affects evolutionary processes within populations. Under adaptive connectivity, gene flow is sufficient to spread advantageous alleles. Under inbreeding connectivity, gene flow is sufficient to avoid the harmful effects of local inbreeding. Under drift connectivity, gene flow is sufficient to maintain similar allele frequencies among populations. Redrawn from Lowe and Allendorf [80].

Figure IB. The interplay of selection, population size, and gene flow. The expected influence of effective population size $\left(N_{\mathrm{e}}\right)$ on the relationship between migration rate $(\mathrm{m})$ and mean fitness in a population exposed to strong directional selection. We define mean fitness according to population genetics theory, as the sum of the fitnesses of genotypes in the population weighted by their proportions [88]. A comprehensive measure of realized mean fitness in natural populations should incorporate a range of other ecological, demographic, and life history factors (i.e., many of the factors addressed in this paper; Figure 1), as others have noted [100]. However, the relationship described here will, nevertheless, underlie population response to those factors. 


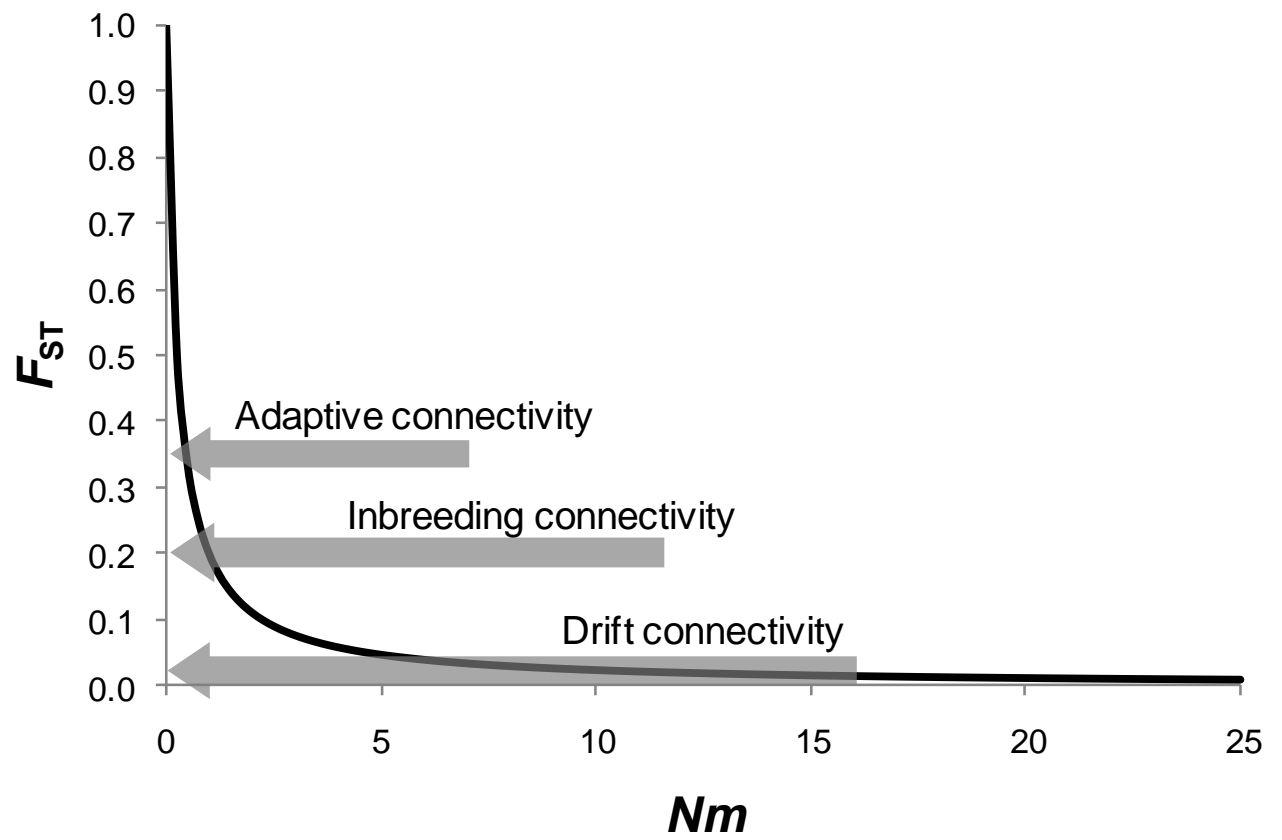

Figure IA 


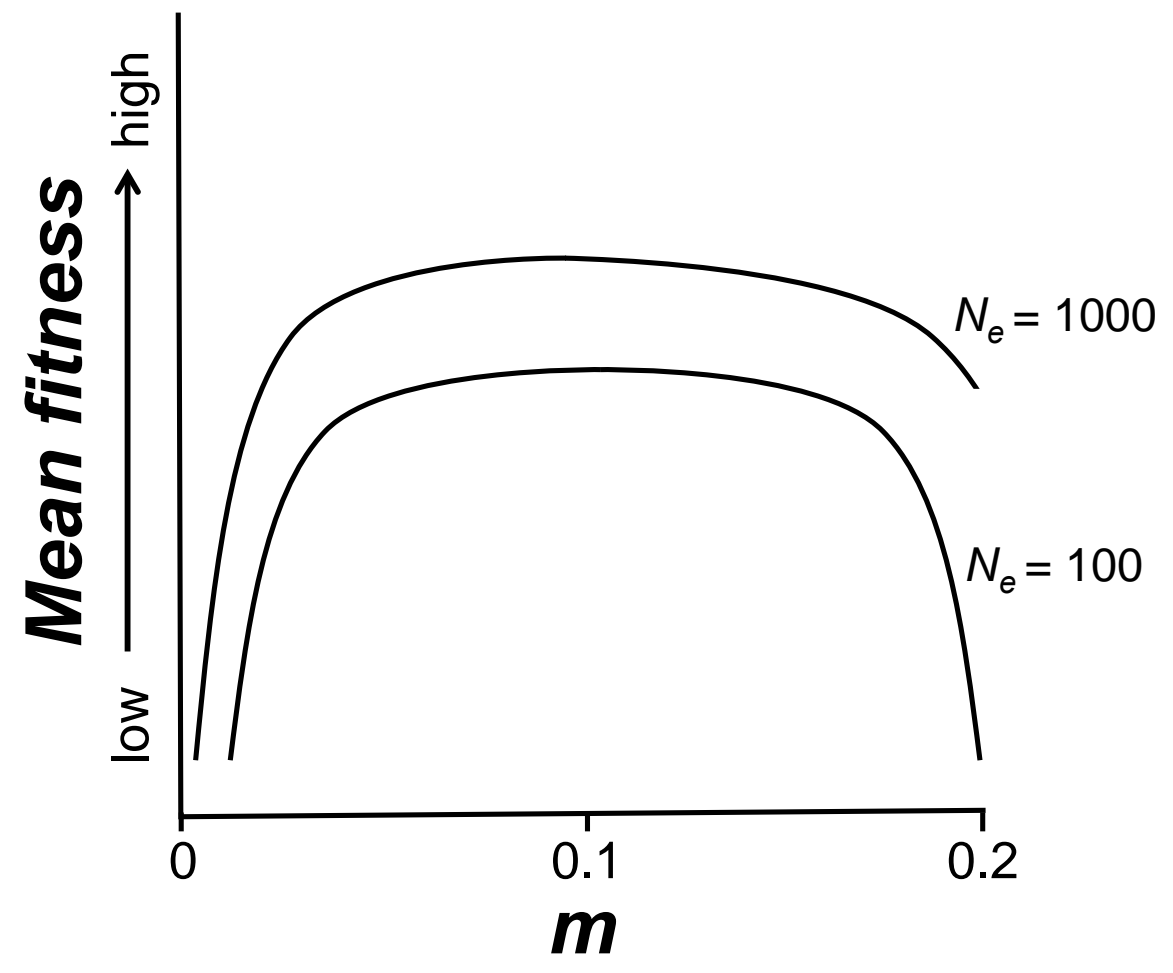

Figure IB 


\section{Glossary}

Compensatory mortality: when one source of mortality largely replaces another source of mortality, resulting in little or no change in population dynamics.

Density dependence: when population growth or specific demographic rates (e.g., mortality, fecundity) are regulated by the density of the population.

Dispersal: permanent movement away from an origin and long-term settlement at a new location.

Disruptive selection: natural selection that favors extreme values of a trait over intermediate values, also known as diversifying selection.

Eco-evolutionary dynamics: interplay between ecological and evolutionary dynamics in real time (i.e., relatively instantaneously).

Effective population size $\left(N_{e}\right)$ : size of an ideal population that would experience the same amount of genetic drift as the observed population.

Fixation index $\left(\boldsymbol{F}_{\mathrm{ST}}\right)$ : a measure of population subdivision that indicates the proportion of heterozygosity found between populations relative to the amount within populations. 
Gene flow: movement of genes from one population to another. In population genetics theory, gene flow is represented by migration rate $(m)$, the proportion of individuals in a focal population that are immigrants.

Genetic drift: change in gene frequencies over time due to random differences in the survival and fecundity of individuals, as well as binomial sampling of alleles during meiosis.

Genetic rescue: increase in population growth of small populations following immigration, resulting from the reduction of genetic load caused by inbreeding depression.

Hard selection: natural selection that removes from the population individuals whose phenotype does not attain a certain threshold, independent of population density or genotype/phenotype frequency. Hard selection can result in extra mortality, and can therefore depress population size.

Selection coefficient: a measure of the reduction in the relative fitness of a given genotype. The selection coefficient takes a value between zero (no reduction in fitness) and one (fitness is zero). 
Soft selection: natural selection that eliminates individuals that do not achieve a certain relative value of a given trait. Under soft selection, selective deaths are substituted for non-selective mortality so it has little effect on population size. 


\section{References}

1. Mills, L.S. (2013) Conservation of wildlife populations: demography, genetics, and management. Wiley-Blackwell

2. Saccheri, I. and Hanski, I. (2006) Natural selection and population dynamics. Trends Ecol. Evol. 21, 341-347

3. Allendorf, F.W., et al. (2013) Conservation and the genetics of populations (2nd edn). Wiley-Blackwell

4. Roughgarden, J.E. (1979) Theory of population genetics and evolutionary ecology. Macmillan

5. Kokko, H. and Lopez-Sepulcre, A. (2007) The ecogenetic link between demography and evolution: can we bridge the gap between theory and data? Ecol. Lett. 10, 773-782

6. Lanfear, R., et al. (2014) Population size and the rate of evolution. Trends Ecol. Evol. $29,33-41$

7. Slatkin, M. (1985) Gene flow in natural populations. Annu. Rev. Ecol. Syst. 16, 393430

8. Reznick, D. (2016) Hard and soft selection revisited: how evolution by natural selection works in the real world. J. Hered. 107, 3-14

9. Crow, J.F. and Kimura, M. (1970) An introduction to population genetics theory. The Blackburn Press

10. Pelletier, F., et al. (2009) Eco-evolutionary dynamics. Philos. T. R. Soc. 364, 14831489

11. Hendry, A.P. (2016) Eco-evolutionary dynamics. Princeton University Press 
12. Siepielski, A.M., et al. (2016) Experimental evidence for an eco-evolutionary coupling between local adaptation and intraspecific competition. Am. Nat. 187, 447-456 13. Agrawal, A.A., et al. (2013) A field experiment demonstrating plant life-history evolution and its eco-evolutionary feedback to seed predator populations. Am. Nat. 181, S35-S45

14. Williams, J.L., et al. (2016) Rapid evolution accelerates plant population spread in fragmented experimental landscapes. Science 353, 482-485

15. Travis, J., et al. (2014) Do eco-evo feedbacks help us understand nature? Answers from studies of the Trinidadian guppy. Adv. Ecol. Res. 50, 1-40

16. Grant, P.R. and Grant, B.R. (2006) Evolution of character displacement in Darwin's finches. Science 313, 224-226

17. Losos, J.B., et al. (2006) Adaptation, speciation, and convergence: a hierarchical analysis of adaptive radiation in Caribbean Anolis lizards. Ann. Mo. Bot. Gard. 93, 24-33 18. Antonovics, J. and Bradshaw, A.D. (1970) Evolution in closely adjacent plant populations VIII. Clinal patterns at a mine boundary. Heredity 25, 349-362

19. Kinnison, M.T. and Hairston, N.G. (2007) Eco-evolutionary conservation biology: contemporary evolution and the dynamics of persistence. Funct. Ecol. 21, 444-454

20. Darwin, C. (1859) The origin of species by means of natural selection. John Murray

21. Crow, J.F. (2008) Commentary: Haldane and beanbag genetics. Int. J. Epidemiol. 37, $442-445$

22. Mayr, E. (1959) Where are we? Cold Spring Harbor Symp. Quant. Biol. 24, 1-14

23. Haldane, J.B.S. (1957) The cost of natural selection. J. Genet. 55, 511-524

24. Wallace, B. (1975) Hard and soft selection revisited. Evolution 29, 465-473 
25. Charlesworth, B. (2013) Why we are not dead one hundred times over. Evolution 67, $3354-3361$

26. Hendry, A.P. (2013) Key questions in the genetics and genomics of eco-evolutionary dynamics. Heredity 111, 456-466

27. Ehrlich, P.R. and Raven, P.H. (1969) Differentiation of populations. Science 165, $1228-1232$

28. Urban, M.C. (2011) The evolution of species interactions across natural landscapes. Ecol. Lett. 14, 723-732

29. Thompson, J.N. (2005) The geographic mosaic of coevolution. The University of Chicago Press

30. Rasanen, K. and Hendry, A.P. (2008) Disentangling interactions between adaptive divergence and gene flow when ecology drives diversification. Ecol. Lett. 11, 624-636 31. Richardson, J.L., et al. (2014) Microgeographic adaptation and the spatial scale of evolution. Trends Ecol. Evol. 29, 165-176

32. Rees, M. and Ellner, S.P. (2016) Evolving integral projection models: evolutionary demography meets eco-evolutionary dynamics. Methods Ecol. Evol. 7, 157-170

33. Hanski, I., et al. (2011) Eco-evolutionary metapopulation dynamics and the spatial scale of adaptation. Am. Nat. 177, 29-43

34. Darwin, C. (1876) The effects of cross and selffertilization in the vegetable kingdom. J. Murray and Co.

35. Johnson, H.E., et al. (2011) Translating effects of inbreeding depression on component vital rates to overall population growth in endangered bighorn sheep. Conserv. Biol. 25, 1240-1249 
36. Bolnick, D.I., et al. (2011) Why intraspecific trait variation matters in community ecology. Trends Ecol. Evol. 26, 183-192

37. Kendall, B.E. and Fox, G.A. (2002) Variation among individuals and reduced demographic stochasticity. Conserv. Biol. 16, 109-116

38. Morton, N.E., et al. (1956) An estimate of the mutational damage in man from data on consanguineous marriages. Proc. Natl. Acad. Sci. U. S. A. 42, 855-863

39. Grueber, C.E., et al. (2010) Inbreeding depression accumulation across life-history stages of the endangered takahe. Conserv. Biol. 24, 1617-1625

40. Kardos, M., et al. (2015) Measuring individual inbreeding in the age of genomics: marker-based measures are better than pedigrees. Heredity 115, 63-72

41. Bensch, S., et al. (2006) Selection for heterozygosity gives hope to a wild population of inbred wolves. Plos One 1

42. Vrijenhoek, R.C. (1989) Genotypic diversity and coexistence among sexual and clonal forms of Poeciliopsis. In Speciation and its consequences (Otte, D. and Endler, J.A., eds), pp. 386-400, Sinauer Associates

43. Lively, C.M., et al. (1990) Red Queen hypothesis supported by parasitism in sexual and clonal fish. Nature 344, 864-866

44. Frankham, R. (2015) Genetic rescue of small inbred populations: meta-analysis reveals large and consistent benefits of gene flow. Mol. Ecol. 24, 2610-2618 45. Hedrick, P.W., et al. (2014) Genetic rescue in Isle Royale wolves: genetic analysis and the collapse of the population. Conserv. Genet. 15, 1111-1121

46. Peterson, R.O., et al. (2014) Trophic cascades in a multicausal world: Isle Royale and Yellowstone. Annu. Rev. Ecol. Syst. 45, 325-345 
47. Allendorf, F.W. (1986) Genetic drift and the loss of alleles versus heterozygosity. Zoo Biol. 5, 181-190

48. Richards, A.J. (1986) Plant breeding systems. Allend and Unwin

49. Wright, S. (1960) On the number of self-incompatibility alleles maintained in equilibrium by a given mutation rate in a population of given size - a reexamination.

Biometrics 16, 61-85

50. Reinartz, J.A. and Les, D.H. (1994) Bottleneck-induced dissolution of selfincompatibility and breeding system consequences in Aster furcatus (Asteraceae). Am. J. Bot. 81, 446-455

51. Crozier, R.H. (1971) Heterozygosity and sex determination in haplo-diploidy. Am. Nat. 105, 399-\&

52. Hedrick, P.W., et al. (2006) Genetic sex determination and extinction. Trends Ecol. Evol. 21, 55-57

53. Brook, B.W. and Bradshaw, C.J.A. (2006) Strength of evidence for density dependence in abundance time series of 1198 species. Ecology 87, 1445-1451

54. Sæther, B.E. and Engen, S. (2015) The concept of fitness in fluctuating environments. Trends Ecol. Evol. 30, 273-281

55. Reed, T.E., et al. (2013) Population growth in a wild bird is buffered against phenological mismatch. Science 340, 488-491

56. Holt, R.D. (1990) The microevolutionary consequences of climate change. Trends Ecol. Evol. 5, 311-315

57. Gharrett, A.J., et al. (2013) Fine-scale temporal adaptation within a salmonid population: mechanism and consequences. Mol. Ecol. 22, 4457-4469 
58. Fisher, R.A. (1930) The genetical theory of natural selection. Oxford University Press

59. Araki, H., et al. (2007) Effective population size of steelhead trout: influence of variance in reproductive success, hatchery programs, and genetic compensation between life-history forms. Mol. Ecol. 16, 953-966

60. Allendorf, F.W., et al. (2008) Genetic effects of harvest on wild animal populations. Trends Ecol. Evol. 23, 327-337

61. Lenormand, T. (2002) Gene flow and the limits to natural selection. Trends Ecol. Evol. 17, 183-189

62. Fitzpatrick, S.W., et al. (2014) Locally adapted traits maintained in the face of high gene flow. Ecol. Lett. 18, 37-47

63. Edelaar, P. and Bolnick, D.I. (2012) Non-random gene flow: an underappreciated force in evolution and ecology. Trends Ecol. Evol. 27, 659-665

64. Lowe, W.H. and McPeek, M.A. (2014) Is dispersal neutral? Trends Ecol. Evol. 29, $444-450$

65. Fraser, D.F., et al. (2001) Explaining leptokurtic movement distributions: intrapopulation variation in boldness and exploration. Am. Nat. 158, 124-135 66. Sih, A., et al. (2012) Ecological implications of behavioural syndromes. Ecol. Lett. $15,278-289$

67. van Oers, K., et al. (2004) A genetic analysis of avian personality traits: correlated response to artificial selection. Behav. Genet. 34, 611-619 
68. Duckworth, R.A. and Aguillon, S.M. (2015) Eco-evolutionary dynamics:

investigating multiple causal pathways linking changes in behavior, population density and natural selection. J. Ornithol. 156, S115-S124

69. Duncan, S.I., et al. (2010) Relatedness and genetic structure in a socially polymorphic population of the spider Anelosimus studiosus. Mol. Ecol. 19, 810-818 70. Pruitt, J.N. and Modlmeier, A.P. (2015) Animal personality in a foundation species drives community divergence and collapse in the wild. J. Anim. Ecol. 84, 1461-1468 71. Pruitt, J.N. and Goodnight, C.J. (2014) Site-specific group selection drives locally adapted group compositions. Nature 514, 359-362

72. Canestrelli, D., et al. (2016) The tangled evolutionary legacies of range expansion and hybridization. Trends Ecol. Evol. 31, 677-688

73. Cote, J., et al. (2010) Personality traits and dispersal tendency in the invasive mosquitofish (Gambusia affinis). P. R. Soc. B 277, 1571-1579

74. Hanski, I. and Mononen, T. (2011) Eco-evolutionary dynamics of dispersal in spatially heterogeneous environments. Ecol. Lett. 14, 1025-1034

75. Shine, R., et al. (2011) An evolutionary process that assembles phenotypes through space rather than through time. Proc. Natl. Acad. Sci. U.S.A. 108, 5708-5711

76. Coulson, T., et al. (In review) Quantitative genetics meets integral projection models: unification of widely used methods from ecology and evolution.

77. Van Straalen, N.M. and Roelofs, D. (2012) An introduction to ecological genomics. Oxford University Press

78. Myburg, A.A., et al. (2014) The genome of Eucalyptus grandis. Nature 510, 356-362 79. Royle, J.A., et al. (2014) Spatial capture-recapture. Elsevier 
80. Lowe, W.H. and Allendorf, F.W. (2010) What can genetics tell us about population connectivity? Mol. Ecol. 19, 3038-3051

81. Endler, J.A. (1986) Natural selection in the wild. Princeton University Press 82. Stelkens, R.B., et al. (2012) Genetic and phenotypic population divergence on a microgeographic scale in brown trout. Mol. Ecol. 21, 2896-2915

83. Cubaynes, S., et al. (2012) Testing hypotheses in evolutionary ecology with imperfect detection: capture-recapture structural equation modeling. Ecology 93, 248-255 84. Cam, E. (2008) Contribution of capture-mark-recapture modeling to studies of evolution by natural selection. In Modeling demographic processes in marked populations (Thomson, D.L., et al., eds), pp. 83-130, Springer

85. McDevitt, A.D., et al. (2013) Individual variation in dispersal associated with phenotype influences fine-scale genetic structure in weasels. Conserv. Genet. 14, 499-509 86. Lohr, J.N. and Haag, C.R. (2015) Genetic load, inbreeding depression, and hybrid vigor covary with population size: an empirical evaluation of theoretical predictions. Evolution 69, 3109-3122

87. Wood, C.W. and Brodie, E.D. (2016) Evolutionary response when selection and genetic variation covary across environments. Ecol. Lett. 19, 1189-1200

88. Wright, S. (1969) Evolution and the Genetics of Populations, Volume 2. University of Chicago Press

89. Haldane, J.B.S. (1990) The causes of evolution. Princeton University Press 90. Parvinen, K. (2005) Evolutionary suicide. Acta Biotheor. 53, 241-264 
91. Johansson, M., et al. (2007) Does habitat fragmentation reduce fitness and adaptability? A case study of the common frog (Rana temporaria). Mol. Ecol. 16, 26932700

92. Li, W.H. (1978) Maintenance of genetic variability under the joint effect of mutation, selection and random drift. Genetics $90,349-382$

93. Wade, M.J. and Goodnight, C.J. (1998) Perspective: The theories of Fisher and Wright in the context of metapopulations: When nature does many small experiments. Evolution 52, 1537-1553

94. Wright, S. (1931) Evolution in mendelian populations. Genetics 16, 97-159

95. Lynch, M., et al. (1993) The mutational meltdown in asexual populations. J. Hered. $84,339-344$

96. Mills, L.S. and Allendorf, F.W. (1996) The one-migrant-per-generation rule in conservation and management. Conserv. Biol. 10, 1509-1518

97. Waples, R.S. and Gaggiotti, O. (2006) What is a population? An empirical evaluation of some genetic methods for identifying the number of gene pools and their degree of connectivity. Mol. Ecol. 15, 1419-1439

98. Rieseberg, L.H. and Burke, J.M. (2001) A genic view of species integration. J. Evol. Biol. 14, 883-886

99. Wright, S. (1951) The genetical structure of natural populations. Ann. Eugen. 15, $323-354$

100. Lande, R., et al. (2009) An evolutionary maximum principle for density-dependent population dynamics in a fluctuating environment. Philos. T. R. Soc. B 364, 1511-1518 